\title{
Cryo - Focused Ion Beam preparation of biological materials for retrieval and examination by cryo - TEM.
}

\author{
E.J.Basgall* and D. Nicastro** \\ *Nanofabrication Facility, The Pennsylvania State University, 192 MRI Bldg, 203 Innovation Blvd, \\ University Park, PA 16802; \\ **Boulder Laboratory for 3-D Electron Microscopy of Cells, University of Colorado, Campus Box \\ 347, Boulder, CO 80309
}

Vitrification of cells and tissues by rapid freezing preserves their structures in a near-to native state and to a fine level of detail [1]. Vitreous ice is amorphous without destructive ice crystals, however, to avoid crystal formation the cooling rate has to be very high and thus freezing techniques like plunge-freezing are limited to relatively thin specimen $(5-10 \mu \mathrm{m})$. High pressure freezing (HPF) on the other hand, has allowed reproducible near vitrification of biological samples of up to $300 \mu \mathrm{m}$ thickness [2]. To generate slices of appropriate thickness for cryo-TEM from frozen-hydrated (FH) samples that are not intrinsically thin enough $(<\sim 500 \mathrm{~nm})$, cryo-sectioning with diamond knives has been used [3]. However, cryo-microtomy of FH biological materials has always been problematic and the FH sections suffer from artifacts, such as compression along the cutting direction, crevasses, knife marks and chatters [for review see 4].

Recent technological advances in the form of a focused ion beam (FIB) column combined with a scanning electron optical column (dual beam FIB) allow for ion beam cross sectioning and imaging with the same instrument. Sections and cylinders have been produced at ambient temperature, and lifted out after FIB milling for subsequent TEM analysis [5]. The FIB tool utilizes a liquid metal ion gun to produce an energetic stream of Ga-ions. This stream of heavy ions is finely focused and used to erode away selected areas of material of a specimen within the sample chamber. The addition of a cryo-transfer system, preparative chamber and cryo-stage unit to dual beam FIB instruments has opened up the possibility to use "cryo-fibbing" as an alternative approach for the production of thin FH samples [6,7]. We have begun to develop the techniques and procedures necessary to introduce FH material into the FIB, generate specific milling pattern (Fig. A) under visual control, retrieve the manipulated samples and transfer the cut material onto TEM grids for evaluation by cryo-TEM imaging.

Test samples of bacteria and fission yeast were prepared by HPF at the Boulder facility and transferred in a dry cryo-shipper to the PSU Nanofabrication facility for cryo-FIB. Samples were held under liquid nitrogen at all times up until transferring into the cryo-prep chamber airlock. An aluminum SEM stub was modified (Fig. B) in order to hold the HPF carriers securely during the transfer, milling and retrieval stages. Any ice contamination on the specimen surface that may have formed during the transfer step was removed by scraping with the cooled cryo-knife inside the evacuated prep chamber. Using cryo-FIB milling, areas of cells and water are progressively removed until a vertical wall section remains (Figs. C,D). Cylinders and square post shapes can also be milled (Fig. E). Though our results are preliminary, this technique holds great promise for obtaining cryo-sections for subsequent cryo-TEM analysis. 
References:

[1] J. Dubochet, et al., Quaterly Review of Biophysics 21 (1988) 129-228. [2] M. Müller and H. Moor, In: The Science of Biological Specimen Preparation, Proc. of the 2nd Pfefferkorn Conference, O'Hare, IL, USA. p.131-138, 1984. [3] A. W. McDowall, et al., J. Microsc., 131 (1983) 1-9. [4] A. Al-Amoudi, et al., J. Struct. Biol., 150 (2005) 109-121. [5] L.A.Gianuzzi, Microsc Today, 12 (2004) 34-35. [6] H. Mulders, G.I.T. Imaging and Microscopy, 5 (2003) 8. [7] M. Marko, et al., Microsc. Microanal. 11(Suppl 2) (2005) 802.

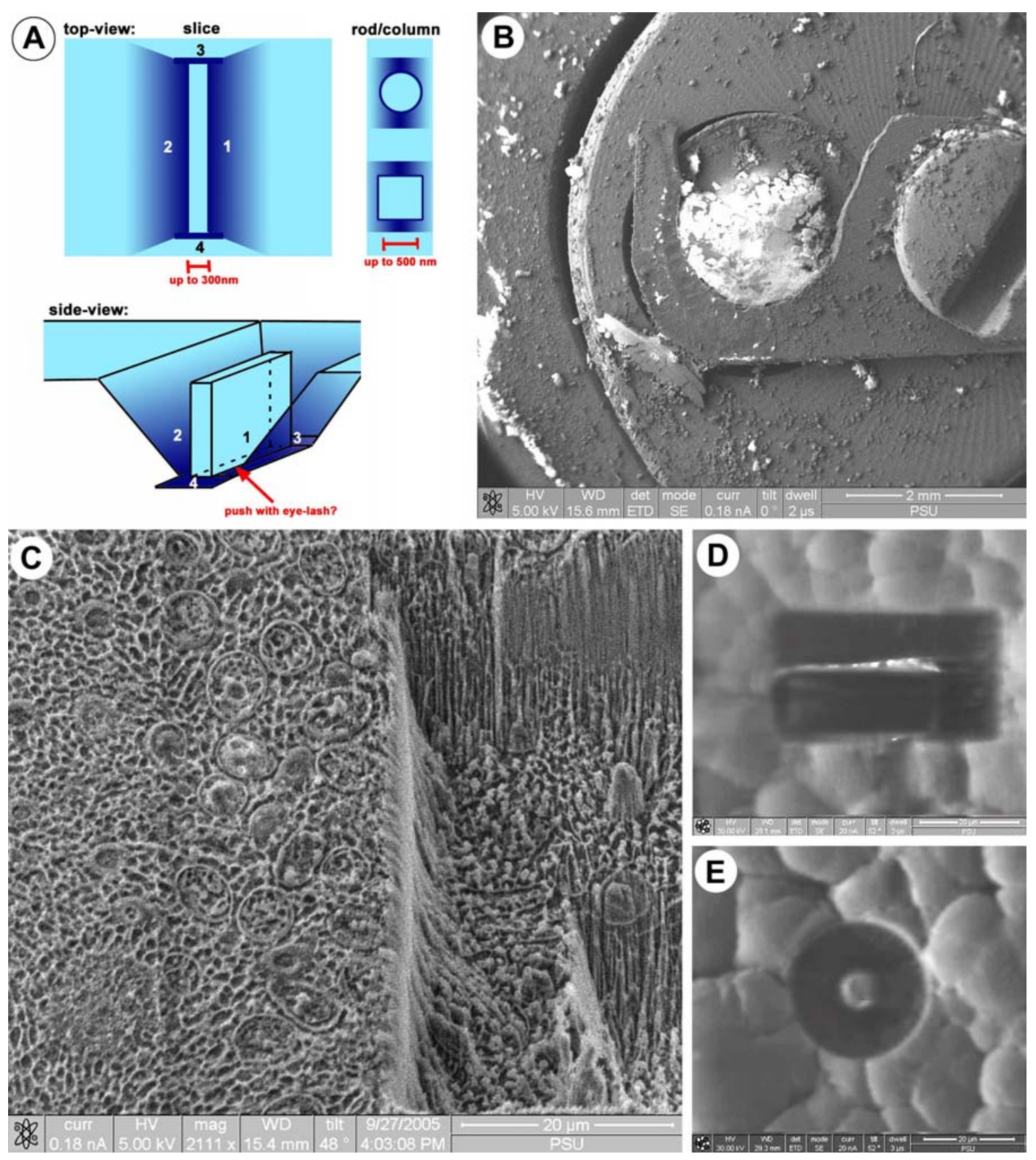

Fig. A) Milling scheme for cryo-FIB on biological material. B) Modified SEM stub with well and HPF carrier holding latch. C) Preliminary cryo-FIB on plunge-frozen yeast cells. D,E) Milling of a FH TEM sample in form of a thin slab (D) and a cylinder (E), respectively. 